\title{
Cone Beam Computed Tomography in Orthodontics
}

\author{
Furkan Dindaroğlu, Enver Yetkiner
}

Department of Orthodontics, Ege University School of Dentistry, Izmir, Turkey

\begin{abstract}
The advent of three-dimensional (3D) imaging technology has caused a significant change in the diagnostic approach practiced in dentistry, and in particular, orthodontics. Although conventional imaging methods such as orthopantomography and lateral cephalometric and anteroposterior graphs provide sufficient information in mild to moderate orthodontic anomalies, 3D imaging can be a necessity in severe skeletal anomalies or tooth impactions. Computed tomography (CT) has been frequently used when detailed 3D imaging is necessary despite its relatively high cost, low vertical resolution, and high dose of radiation. In contrast to conventional CT application, the development of cone beam computed tomography (CBCT) technology has had important advantages over the conventional method, such as minimization of the radiation dose, image accuracy, rapid scan time, fewer image artifacts, chair-side image display, and real-time analysis. These advantages have provided dental practitioners the opportunity to benefit more frequently from 3D imaging by relatively diminishing radiation dose considerations, financial burden, and availability, in particular. Therefore, the aim of this review is to highlight the current understanding of CBCT practice in orthodontics and to summarize clinically relevant conditions.
\end{abstract}

Keywords: Cone beam computed tomography, orthodontics, 3D imaging

\section{Orthodontics and Radiographic Imaging}

The primary aim of orthodontic treatment, and in particular, dentofacial orthopedics, is the correction of malocclusions and facial discrepancies, which are related to dental and skeletal divergences. The treatment plan to achieve this aim is usually based on a patient's main complaint as well as the capabilities of orthodontics. Expected treatment outcomes have esthetic, psychosocial, and functional aspects. Differential diagnosis and treatment planning of such anomalies usually require detailed radiographic imaging of facial components. Conventional radiography and lateral cephalometric imaging, in particular, have formed the cornerstone of the diagnostic procedure for decades. ${ }^{1}$ The limitations of such conventional techniques arising from the alteration of three-dimensional (3D) anatomy to two-dimensional images have been pronounced as one of the factors inducing treatment failure and relapse. ${ }^{2}$ These include magnification differences, geometric distortion, superimposition of anatomic structures, projective displacements of anatomic structures, rotational errors, and linear projective transformations. ${ }^{3}$ With the advent of computed tomography (CT), and more recently, cone beam computed tomography (CBCT), 3D imaging of the anatomy can be achieved, which enables the visualization of complex relations of adjacent tissues. ${ }^{4}$ This has been enhanced with the development of computer software technology, which has enabled detailed assessment of these images for varying demands.

\section{New Demands in Orthodontic Radiography}

Radiographs that are requested by the orthodontist are usually used as an adjunct to clinical diagnosis and assessment of treatment effects and outcome. However, the justification of these radiographs for each patient is extremely crucial depending on the patient's primary complaint, patient history, clinical inspection, and possible treatment objectives. ${ }^{5}$ The main aspects of justified radiography practice are deciding on the most appropriate imaging technique, obtaining the image, and interpretation. The most appropriate imaging technique should have minimum radiation exposure and maximum diagnostic benefit. Still, this practice remains controversial even in countries where guidelines define algorithms based on patient age and clinical findings. Routine ordering of radiographs for all patients is strictly classified as contraindicated in the European Guidelines on Dental 
Radiology ${ }^{6}$ as well as the guideline accepted in the United Kingdom. ${ }^{7}$ Interestingly, there is no applied guideline in the United States, although the American Dental Association Council on Scientific Affairs has recently issued an advisory statement on the use of $C B C T$ in dentistry. ${ }^{8}$

Cone beam computed tomography has entered the area of interest in many orthodontic and pediatric dentistry applications. ${ }^{9-11}$ There are two main novelties that are introduced to clinical orthodontic practice with the emerging CBCT technology. The most striking advantage for the clinician is the possibility of viewing facial anatomic structures and the dentition in 3D from different angles. This is even more enhanced with the help of different computer software programs, letting the clinician measure area and volume of anatomical structures, such as the airway volume and facial outline. The second advantage is the possibility of obtaining conventional images such as panoramic radiograph and lateral cephalogram from a single CBCT scan. However, this can be considered as an advantage when ordered prior to any conventional imaging in terms of reducing radiation exposure, which is not always the case. At present, the guidelines that are in function in Europe have not integrated this new technology into their algorithm.

Cone beam computed tomography imaging has been demonstrated to provide clinical efficacy in altering treatment planning for impacted maxillary canines, ${ }^{12,13}$ unerupted teeth, presence of root resorption, and severe skeletal discrepancies. ${ }^{12}$ This potential of additional diagnostic information and precision of clinical decision has led some clinicians and innovators to advocate the replacement of conventional imaging modalities with CBCT for routine orthodontic diagnosis and treatment ${ }^{9,14,15}$ However, no evidence-based benefit has been demonstrated for patients specifically referred for abnormalities of the temporomandibular joint, airway assessment, or dental crowding. ${ }^{12}$

\section{Knowledge on CBCT Imaging: Orthodontist and Patient}

Because the CBCT technology is relatively new and rapidly developing, it is rather difficult for the clinician to follow emerging developments as well as to deepen the clinical experience. However, the orthodontist should be very well informed of the recent innovations regarding this technology to be able to inform the patient regarding the risks of radiation exposure as well as fulfil the necessary diagnostic approach. The information obtained from $C B C T$ imaging requires a substantial level of expertise for correct and precise interpretation. In other words, clinicians with inadequate experience are in danger of misinterpreting CBCT images, resulting in overlooked or false-positive diagnoses. ${ }^{10}$ The prevention of this risk only becomes possible when the orthodontist is regularly being updated with the necessary knowledge and clinical experience is improved.

Although there is inadequate evidence available to justify the use of CBCT in routine orthodontic cases, some conditions where $\mathrm{CBCT}$ may be beneficial regarding a valued benefit-to-risk assessment could be defined. ${ }^{7}$ Incidental findings in CBCT images of orthodontic patients are common, ${ }^{16-18}$ and some are critical to patient health. ${ }^{19}$ Clinicians who order or perform CBCT for orthodontic patients are responsible for interpreting the entire image volumes, just as they are responsible for interpreting all regions of other radiographic images that they order. ${ }^{20}$

The frequency of incidental discoveries in CBCT images irrelevant to the original purpose of the scan has been reported to be as high as $25 \% .^{18} \mathrm{An}$ important question that requires further investigation is the capability of the orthodontist to identify non-orthodontic-relevant findings and to make appropriate referrals when required. In contrast, the potential for inadvertent diagnosis of false-positive findings may add to unnecessary costs as well as cause unnecessary anxiety to the patient and family. In a recent study, it was shown that orthodontists and orthodontic residents miss approximately $67 \%$ of lesions and have a $50 \%$ false-positive detection rate in CBCT images. ${ }^{21}$

Based on these considerations, the following recommendations are related to performing and interpreting $C B C T$ studies:

1. Clinicians have an obligation to attain and improve their professional skills through a lifelong learning process to perform successful CBCT examinations as well as accurate interpretations. Clinicians need to attend continuing education courses to maintain familiarity with the technical and operational aspects of CBCT and to maintain current knowledge of scientific advances and health risks associated with the use of CBCT.

2. Clinicians have legal responsibilities when operating CВCT equipment and interpreting images and are expected to comply with all regulations.

3. It is important that patients/guardians know about the limitations of CBCT with regard to the visualization of soft tissues, artifacts, and noise.

\section{Radiation Dose Considerations}

The decision for any radiographic imaging procedure should be performed following the as-low-as-reasonably-achievable (ALARA) principle. CBCT can be justified only if the anticipated information does have the potential to change a patient's treatment modalities or outcome. Radiation risk has most often been estimated by calculating the effective dose ${ }^{22}$ of a CBCT scan and comparing this value to the following: 1) measurements obtained from comparable imaging modalities (e.g., multiples of typical panoramic images or a multi-slice medical (T), 2) background equivalent radiation time (e.g., days of background), or 3) radiation detriment (stochastic-cancer rate). Often, the base unit of these comparisons (typical panoramic dose, background radiation, weighted probabilities of fatal and nonfatal cancers) is variable and not absolute. This implies that depending on the panoramic image dose used for the comparison, the risk of CBCT may be reported either conservatively or liberally compared with the risk of panoramic radiography. Because $\mathrm{CBCT}$ exposes patients to ionizing radiation that may pose elevated risks to some high-risk patients (pregnant or younger patients), elaborate explanations and disclosure to patients about radiation exposure risks, benefits, and imaging modality alternatives are crucial and should be documented in the patient records. ${ }^{8}$ 
Radiation exposure and comparisons between CBCT and other forms of imaging: CBCT has substantially lower radiation but lower resolution than spiral $C T{ }^{23}$ The currently available $\mathrm{CBCT}$ units have variable radiation exposure in the range of 11 to $674 \mu \mathrm{Sv}$ for dento-alveolar scans and 30 to $1073 \mu \mathrm{Sv}$ for full craniofacial scans. Besides, the radiation dose for multislice CT ranges between 280 and $1410 \mu S_{v}{ }^{24}$ The approximate radiation doses for conventional imaging are as follows: a panoramic radiograph, 2.7-24.3 $\mu \mathrm{Sv}$; a lateral cephalogam, $<6 \mu \mathrm{Sv}$; and a full mouth series, $13-100 \mu \mathrm{Sv}^{24}$

Comparison of CBCT vs. panoramic radiography: $A$ subjective comparison of images from two different CBCT units (NewTom 9000, QR s.r.l.; Verona, Italy and Arcadis Orbic 3D, Siemens Medical Solutions; Erlangen, Germany) and routine panoramic radiography showed that CBCT can provide more information than conventional radiographs in localizing impacted and retained teeth, root resorption, cleft lip and palate (CLP) evaluation, and third molar evaluations but not for evaluating changes in TMJ. ${ }^{25}$

lonizing radiation creates either a deterministic effect that causes the death of cells or a stochastic effect that irreversibly alters the cells by damaging cellular DNA, resulting in cancer. A deterministic effect occurs with a high dose of radiation exposures in rather short periods of time, and clinical changes occur after the limits are reached, which is never the case in conventional oral and maxillofacial radiology. A stochastic effect occurs at the cellular level and is accumulated over time. Therefore, immediate effects are not observed but the risk of carcinogenesis eventually increases as the exposure recurs. Recently, a potential association between intracranial meningioma and dental radiographic procedures has been reported, which raises more attention on this particular topic. ${ }^{26}$ The role of low-dose radiographic procedures in the risk of carcinogenesis is not easy to assess. There is no evidence indicating the initiation of carcinogenesis due to dental radiographic procedures, whereas the absence of such damage is also not shown. Therefore, the maximum limit of radiographic exposure cannot be stated with the presence of a potential risk that is critical. Hence, the advisory guidelines focus on optimizing the diagnostic radiographic imaging settings to minimize patient risk. ${ }^{8}$

Dose consideration in younger patients: An effective dose is the relative susceptibility of different tissues to the same amount of radiation exposure. The risk of carcinogenesis in specific tissues and organs varies depending on risk-weighting factors (differences in exposed tissue sensitivity, gender, and age) and absorbed radiation dose. The effective dose is calculated by multiplying organ doses by risk-weighting factors. ${ }^{22}$

Young children are much more susceptible to the stochastic effect of ionizing radiation than adolescents and adults because of their extremely high cellular reproduction and organ development. In particular, specific organs and effective doses of particular organs in the craniofacial region such as salivary glands are $30 \%$ higher than for adolescents. ${ }^{27}$ Furthermore, their relatively longer life expectancy in terms of years increases the risk of a cumulative effect in creating carcinogenesis. Another factor that increases this risk in younger children becomes evident when pediatric exposure reduction techniques are not incorporated while obtaining the CBCT scan, because of which the conventional doses that are minimally accepted for adult patients are exceeded. Thus, the effective dose of CBCT imaging obtained for orthodontic records is of a particular concern because the average age group for orthodontic treatment is the pediatric population. Strikingly, it is predicted that children may be two to 10 times or more prone to radiation-induced carcinogenesis than adults. 22,28

\section{How to Minimize Patient Radiation Exposure}

A justified decision on a CBCT scan does not necessarily eliminate the responsibility of the clinician who has made the request for the settings in which the image is to be obtained. Depending on the radiographic equipment and operator preferences, the radiation doses can be significantly altered. Therefore, exposure (e.g., mA, kV), image quality (e.g., number of basis images, resolution, arc of trajectory) and beam collimation [e.g., field of view (FOV)] settings can be altered. CBCT units from different manufacturers vary in dose by as much as 10-fold for an equivalent FOV examination. In addition, adjustments of exposure factors to improve image quality are available in many CBCT units and can cause as much as sevenfold differences in patient doses. ${ }^{29}$

Field of view may be small (individual teeth or quadrant), medium (both arches, including TMJ), or large (full head). A smaller FOV is used for assessing individual teeth, for example, impacted teeth and root morphology supernumeraries, or sites for the placement of dental implants or TADs. A medium FOV includes the mandible, maxilla, or both and would typically be used when additional information on occlusal relationships, facial asymmetries, or bilateral TMJ evaluations is required or when the condition(s) of interest such as potentially adverse boundary conditions are present in both arches or jaws. The largest FOV includes the whole head and helps clinicians to visualize relationships between skeletal bases and between teeth and skeletal bases as well as significant anomalies in patients requiring orthognathic surgery or those with craniofacial anomalies. ${ }^{30}$

Based on these considerations, the following specific recommendations are made to minimize patient radiation exposure for CBCT in orthodontics:

1. Use a pulsed exposure mode of acquisition, optimize exposure settings ( $\mathrm{mA}, \mathrm{kV})$. Reduce the number of basis projection images, and employ dose reduction protocols (e.g., reduced resolution), when possible.

2. When other factors remain the same, reduce the size of FOV to match the region of interest (ROI); however, the selection of FOV may result in automatic or default changes in other technical factors (e.g., mAs) that should be considered because these concomitant changes can result in an increase in dose.

3. Use patient protective shielding (such as lead torso aprons; consider the use of thyroid shields), when possible (e.g., for maxillary only scan) to minimize exposure to radiosensitive organs outside FOV of exposure.

4. Ensure that all CBCT equipment is properly installed, routinely calibrated and updated, and meets all governmental requirements and regulations. 


\section{Accuracy of CBCT-derived Cephalograms and Measurements vs. Conventional Method}

Cephalograms obtained from CBCT imaging have been shown to have no significant differences in linear and angular measurements relative to traditional cephalograms, ${ }^{23,31}$ whereas measurement errors are lower than those of conventional cephalograms. ${ }^{3}$ 3D measurements of CBCT images can be performed in several visualization modes such as multiplanar (MPR), volume-rendered (VR), and shaded surface display (SSD). ${ }^{32,33}$ Among these, pointto-point measurements performed in the MPR mode are reported to be highly accurate in comparison with physical skull measurements, whereas the surface anatomy measured in VR and SSD modes has been reported to present a measurement error of $2.3 \%$ when compared with that measured with direct physical measurements. ${ }^{33,34}$ The error in VR and SSD display modes results were interpreted to arise from surface contours that were estimated in these modes. With these findings in mind, it may be anticipated that the identification and targeting of landmarks should be performed using the digital imaging and communications in medicine (DICOM) volume in an MPR display mode.

\section{Case Selection}

Some researchers have provided recommendations for ordering CBCT scans based on specific characteristics, including facial asymmetry, sleep apnea, impacted teeth, intent to use dental mini-implants, consideration of rapid maxillary expansion, and persistent TMJ symptoms. ${ }^{4}$ Others have advocated the routine use of CBCT in standard orthodontic diagnosis and treatment planning because of the additional diagnostic information that is potentially available. ${ }^{15}$ The decision to use any imaging modality before orthodontic treatment is justified when there is a reasonable expectation that a radiograph will result in a clinical benefit.

The SEDENTEXCT project of the European Union had the following primary goal: "to acquire key information necessary for sound and scientifically based clinical use of CBCT" and "to use this information to develop evidence-based guidelines dealing with justification, optimization, and referral criteria for users of dental CBCT." The guidelines section dealing with orthodontic diagnosis concludes that "large volume CBCT should not be used routinely for orthodontic diagnosis." The SEDENTEXCT guidelines are based on a systematic review of the literature, thus representing current evidence-based knowledge at a higher confidence level. ${ }^{24}$ The British Orthodontic Society guidelines provide a similar recommendation: "routine use of CBCT even for most cases of impaction of teeth cannot yet be recommended."7 A similar conclusion has been adopted by the American Association of Orthodontists in 2010: "the AAO recognizes that while there may be clinical situations where a CBCT radiograph may be of value, the use of such technology is not routinely required for orthodontic radiography." 35

Impaction: Impacted and transposed teeth are possibly the most common reason for the use of CBCT imaging in orthodontics. ${ }^{36-38}$ The derived information can enhance the ability to localize impacted or transposed teeth, identify pathological conditions and root resorption, help plan surgical access and bond placement, and define the optimal and most efficient path for extrusion into the oral cavity that avoids or minimizes collateral damage. ${ }^{36-38}$

Relative to traditional radiographs, CBCT scans enable more sensitive and definitive diagnosis of root resorption associated or unassociated with impacted teeth. For root resorption resulting from impacted teeth, CBCT scans provide better visualization of roots than routine radiographs, which can have artifacts owing to the superimposition of structures and the inability to observe the $3 \mathrm{D}$ root structure from all possible directions. ${ }^{38}$

Tooth-Bone Relationships: For orthodontic treatment purposes, the boundary conditions may be defined as the amount (depth and height) and morphology of the alveolar bone relative to tooth root dimensions, angulation, and spatial position. The complex anatomical boundary conditions may limit or dictate the planned or potential tooth movement as well as the final desired spatial position and angulation of the tooth. Patients with alveolar bone phenotypes that clinically appear too narrow to accommodate significant labiolingual or buccolingual displacements or angulations of teeth, patients with compromised periodontium or gingival anatomy or both, and patients in whom the movement of the tooth or teeth may entail translocation past another tooth or obstruction may benefit from CBCT scans for assessing tooth-bone relationships..$^{10,36}$

\section{Recent Innovations}

As the imaging technology develops, the efforts to improve image quality and radiation exposure reduction have led to new innovations. These include automatic exposure control with photon counting, added filtration, flat panel detectors with greater photon sensitivity, customizable FOV collimation, and variable exposure parameters (e.g., mA, kV) and image quality settings (e.g., scan trajectory options and number of basis images). Nevertheless, such innovations and their reliability in terms of image quality and patient safety should be verified by well-designed studies. The efficacy of CBCT imaging for orthodontic diagnosis and treatment planning in terms of its influence on therapy decisions and patient outcome remains to be an area of interest and needs to be investigated elaborately. ${ }^{39}$ Definitely, these considerations and debates may come to an end when innovations provide the clinician with non-radiation imaging technology for the anatomy. To date, it is the responsibility of the medics involved in the process of image requirement or acquirement. On the other hand, with the driving force of developing technology (segmented volumetric measurements, etc.), accurate effective dose and precise indication may be beneficial for minimizing patient risk and maximizing diagnostic benefit.

\section{CONCLUSIONS}

Still, there is a general consensus on the following aspects of $\mathrm{CBCT}$ use in orthodontic practice:

1. The use of CBCT imaging is justified when the benefits to the diagnosis and/or treatment plan outweigh the potential risks of exposure to radiation in the presence of individual 
anamnesis, clinical inspection, and available radiographs. The decision for any radiographic imaging procedure should be performed following the ALARA principle. CBCT can be justified only if the anticipated information has the potential to change a patient's treatment modalities or outcome.

2. $\mathrm{CBCT}$ can be used when there is a controversy between the adequacy of conventional radiographs and 3D imaging. These cases include cleft palate patients, assessment of unerupted tooth position, identification of root resorption caused by unerupted teeth, and planning orthognathic surgery.

3. Restricting FOV, exposure ( $\mathrm{mA}$ and $\mathrm{kV}$ ), number of basis images, and resolution is necessary for optimum visualization of the area of interest.

4. $\mathrm{CBCT}$ imaging is contraindicated to obtain only a lateral cephalometric and/or panoramic radiograph if $C B C T$ results in a higher radiation exposure.

5. The need of conventional radiographs should be carefully considered if the clinical examination prevails the necessity of a CBCT scan for adequate diagnosis and/or treatment planning in order to prevent multiple imaging.

Peer-review: Externally peer-reviewed.

Author Contributions: Concept - F.D., E.Y.; Design - F.D., E.Y.; Supervision - F.D., E.Y.; Resources - F.D., E.Y.; Materials - F.D., E.Y.; Data Collection and/ or Processing - F.D., E.Y.; Analysis and/or Interpretation - F.D., E.Y.; Literature Search - F.D., E.Y.; Writing Manuscript - F.D., E.Y.; Critical Review - F.D., E.Y.; Other - F.D., E.Y.

Conflict of Interest: No conflict of interest was declared by the authors.

Financial Disclosure: The authors declared that this study has received no financial support.

\section{REFERENCES}

1. Atchison KA, Luke LS, White SC. Contribution of pretreatment radiographs to orthodontists' decision making. Oral Surg Oral Med Oral Pathol 1991; 71: 238-45. [CrossRef]

2. Johnston LE. A few comments on an elegant answer in search of useful questions. Semin Orthod 2011; 17: 13-4. [CrossRef]

3. Adams GL, Gansky SA, Miller AJ, Harrell WE, Jr, Hatcher DC. Comparison between traditional 2-dimensional cephalometry and a 3-dimensional approach on human dry skulls. Am J Orthod Dentofacial Orthop 2004; 126: 397-409. [CrossRef]

4. White SC, Pae EK. Patient image selection criteria for cone beam computed tomography imaging. Semin Orthod 2009; 15: 19-28. [CrossRef]

5. Affairs ADACoS. The use of cone-beam computed tomography in dentistry: an advisory statement from the American Dental Association Council on Scientific Affairs. J Am Dent Assoc 2012; 143: 899-902 [CrossRef].

6. European Commission. Item 4.2 the Developing Dentition in Protection Radiation No. 172. Cone Beam CT for Dental and Maxillofacial Radiology (Evidence-based Guidelines). 2011: 45-56.

7. Isaacson KG, Jones M. Guidelines for the use of radiographs in clinical orthodontics. British Orthodontic Society 1994.

8. American Academy of Oral and Maxillofacial Radiology. Clinical recommendations regarding use of cone beam computed tomography in orthodontics. Position statement by the American Academy of Oral and Maxillofacial Radiology. Oral Surg Oral Med Oral Pathol Oral Radiol 2013; 116: 238-57. [CrossRef]
9. Hechler SL. Cone-beam CT: applications in orthodontics. Dent Clin North Am 2008; 52: 809-23. [CrossRef]

10. Kapila S, Conley RS, Harrell WE. The current status of cone beam computed tomography imaging in orthodontics. Dentomaxillofac Radiol 2011; 40: 24-34. [CrossRef]

11. Mah JK, Huang JC, Choo H. Practical applications of cone-beam computed tomography in orthodontics. J Am Dent Assoc 2010; 141: 7S-13S. [CrossRef]

12. Hodges RJ, Atchison KA, White SC. Impact of cone-beam computed tomography on orthodontic diagnosis and treatment planning. Am J Orthod Dentofacial Orthop 2013; 143: 665-74. [CrossRef]

13. Haney E, Gansky SA, Lee JS, Johnson E, Maki K, Miller AJ, et al. Comparative analysis of traditional radiographs and cone-beam computed tomography volumetric images in the diagnosis and treatment planning of maxillary impacted canines. Am J Orthod Dentofacial Orthop 2010; 137: 590-7. [CrossRef]

14. Larson BE. Cone-beam computed tomography is the imaging technique of choice for comprehensive orthodontic assessment. Northwest Dent 2014; 93: 17-20.

15. Silva MAG, Wolf U, Heinicke F, Bumann A, Visser H, Hirsch E. Conebeam computed tomography for routine orthodontic treatment planning: a radiation dose evaluation. Am J Orthod Dentofacial Orthop 2008; 133: 640.e641-645. [CrossRef]

16. Pazera $P$, Bornstein MM, Pazera A, Sendi P, Katsaros C. Incidental maxillary sinus findings in orthodontic patients: a radiographic analysis using cone-beam computed tomography (CBCT). Orthod Craniofac Res 2011; 14: 17-24. [CrossRef]

17. Drage N, Rogers S, Greenall C, Playle R. Incidental findings on cone beam computed tomography in orthodontic patients. J Orthod 2013; 40: 29-37. [CrossRef]

18. Cha JY, Mah J, Sinclair P. Incidental findings in the maxillofacial area with 3-dimensional cone-beam imaging. Am J Orthod Dentofacial Orthop 2007; 132: 7-14. [CrossRef]

19. Rogers SA, Drage N, Durning P. Incidental findings arising with cone beam computed tomography imaging of the orthodontic patient. Angle Orthod 2011; 81:350-5. [CrossRef]

20. Carter L, Farman AG, Geist J, Scarfe WC, Angelopoulos C, Nair MK, et al. American Academy of Oral and Maxillofacial Radiology executive opinion statement on performing and interpreting diagnostic cone beam computed tomography. Oral Surg Oral Med Oral Pathol Oral Radiol Endod 2008; 106: 561-2.[CrossRef]

21. Ahmed F, Brooks SL, Kapila SD. Efficacy of identifying maxillofacial lesions in cone-beam computed tomographs by orthodontists and orthodontic residents with third-party software. Am J Orthod Dentofacial Orthop 2012; 141: 451-9. [CrossRef]

22. Protection ICoR. 1990 recommendations of the International Commission on Radiological Protection, ICRP publication 60. Ann ICRP 1991; 21: 1-3.

23. Farman AG, Scarfe WC. Development of imaging selection criteria and procedures should precede cephalometric assessment with cone-beam computed tomography. Am J Orthod Dentofacial Orthop 2006; 130: 257-65. [CrossRef]

24. SEDENTEXCT project. Radiation protection: cone beam CT for dental and maxillofacial radiology. Evidence based guidelines 2011.

25. Korbmacher H, Kahl-Nieke B, Schöllchen M, Heiland M. Value of two cone-beam computed tomography systems from an orthodontic point of view. J Orofac Orthop 2007; 68: 278-89. [CrossRef]

26. Claus EB, Calvocoressi L, Bondy ML, Schildkraut JM, Wiemels JL, Wrensch M. Dental x-rays and risk of meningioma. Cancer 2012; 118: 4530-7. [CrossRef]

27. Theodorakou C, Walker A, Horner K, Pauwels R, Bogaerts R, Jacobs $\mathrm{R}$, et al. Estimation of paediatric organ and effective doses from dental cone beam CT using anthropomorphic phantoms. Br J Radiol 2012; 85: 153-60. [CrossRef] 
28. Brenner D, Elliston C, Hall E, Berdon W. Estimated risks of radiation-induced fatal cancer from pediatric CT. AJR Am J Roentgenol 2001; 176: 289-96. [CrossRef]

29. Ludlow JB, Ivanovic M. Comparative dosimetry of dental CBCT devices and 64-slice CT for oral and maxillofacial radiology. Oral Surg Oral Med Oral Pathol Oral Radiol Endod 2008; 106: 106-14. [CrossRef]

30. Scarfe WC, Li Z, Aboelmaaty W, Scott SA, Farman AG. Maxillofacial cone beam computed tomography: essence, elements and steps to interpretation. Aust Dent J 2012; 57: 46-60. [CrossRef]

31. Kumar V, Ludlow J, Soares Cevidanes LH, Mol A. In vivo comparison of conventional and cone beam CT synthesized cephalograms. Angle Orthod 2008; 78: 873-9. [CrossRef]

32. Periago DR, Scarfe WC, Moshiri M, Scheetz JP, Silveira AM, Farman AG. Linear accuracy and reliability of cone beam CT derived 3-dimensional images constructed using an orthodontic volumetric rendering program. Angle Orthod 2008; 78: 387-95. [CrossRef]

33. Stratemann SA, Huang JC, Maki K, Hatcher DC, Miller AJ. Evaluating the mandible with cone-beam computed tomography. Am J Orthod Dentofacial Orthop 2010; 137: 58-70. [CrossRef]
34. Stratemann SA, Huang JC, Maki K, Miller AJ, Hatcher DC. Comparison of cone beam computed tomography imaging with physical measures. Dentomaxillofac Radiol 2008; 37: 80-93. [CrossRef]

35. American Association of Orthodontists. Statement on the role of CBCT in orthodontics (26-10 H). eBulletin; May 7, 2010.

36. Oberoi S, Knueppel S. Three-dimensional assessment of impacted canines and root resorption using cone beam computed tomography. Oral Surg Oral Med Oral Pathol Oral Radiol 2012; 113 : 260-7. [CrossRef]

37. Scarfe WC, Farman AG, Sukovic P. Clinical applications of conebeam computed tomography in dental practice. J Can Dent Assoc 2006; 72: 75-80.

38. Alqerban A, Jacobs R, Lambrechts P, Loozen G, Willems G. Root resorption of the maxillary lateral incisor caused by impacted canine: a literature review. Clin Oral Investig 2009; 13: 247-55. [CrossRef]

39. Fryback DG, Thornbury JR. The efficacy of diagnostic imaging. Med Decis Making 1991; 11: 88-94. [CrossRef] 